\title{
Proposta de modelo Casa de Energia Zero (CEZ) de baixo padrão para Zona Bioclimática 07 com avaliação da viabilidade econômica
}

\section{Proposal of a low standard Zero Energy House model for Bioclimatic Zone 07 with economic feasibility assessment}

\author{
${ }^{1}$ Renata Mansuelo Alves Domingos e ${ }^{2}$ Elaise Gabriel \\ ${ }^{1}$ Mestre em Engenharia Civil pela Universidade Federal de Santa Catarina (mansuelo.alves@gmail.com) \\ ${ }^{2}$ Mestre em Engenharia Civil pela Universidade Federal de Santa Maria (elaisegabriel@gmail.com)
}

\begin{abstract}
RESUMO: Com a crescente preocupação em relação ao consumo dos recursos ambientais e gasto energético surgem também novas pesquisas voltadas à área sustentável da construção civil. Edifícios eficientes vem sendo construídos não só com o intuito de atrair novos investimentos, mas também levando em conta o cunho ambiental. Edifícios de Energia Zero - EEZ contam com a aplicação de diversas estratégias passivas visando a eficiência energética dos mesmos e consequentemente a diminuição da demanda por refrigeração, iluminação, aquecimento, incluindo o uso de equipamentos mais eficientes e produzem a energia que consomem, tendo um balanço nulo. Entretanto, o grande problema enfrentado pelos profissionais é até onde usar medidas de eficiência energética em termos de custo benefício, ou seja, até que ponto é viável investir em estratégias passivas para redução do consumo. Essa análise depende do clima e economia do local em que a edificação será inserida, portanto é essencial haver estudos que subsidiem as decisões tomadas. Em virtude disso foi realizada uma análise da viabilidade econômica de implantação de uma EEZ em regiões de clima quente com auxílio de estratégias bioclimáticas. O estudo foi desenvolvido por meio de simulações computacionais no software DesignBuilder e a viabilidade financeira foi definida pelo cálculo do payback utilizando para isso o Valor Presente Líquido. Os resultados demonstraram um tempo de retorno de investimento de aproximadamente 7 anos, mostrando assim a viabilidade econômica da implantação da mesma. Podendo dessa forma ser implantado por meios de políticas públicas, podendo assim ter-se micro geradores com viabilidade econômica.
\end{abstract}

Palavras Chave: Eficiência energética. Estratégias passivas. Energia Fotovoltaica.

\begin{abstract}
With the growing concern about the consumption of environmental resources and energy expenditure, new research is also emerging on the low impact construction industry. Efficient buildings have been built not only with the aim of attracting new investments, but also taking into account the environmental scope. Zero energy buildings - ZEB rely on the application of several passive strategies aiming at their energy efficiency and consequently the reduction of refrigeration, lighting, heating, demand, including the use of more efficient equipment and produce in order to obtain a zero balance. However, the major problem faced by professionals is how many energy efficiency measures to use in terms of cost-benefit, i.e., to what extent is it feasible to invest in passive strategies to reduce consumption. This analysis depends on the climate and economy of the site where the building will be inserted, so studies that support decision-making are essential. Because of this, an economic feasibility analysis was carried out for the implementation of a ZEB in hot climate regions with the assistance of bioclimatic strategies. The study was developed through computational simulations in the DesignBuilder software and financial viability was defined by the payback calculation using the Net Present Value. The results showed an investment return time of approximately 7 years, thus showing the economic viability of its implementation. It can be deployed by means of public policies, thus allowing micro-generators to be economically viable.
\end{abstract}

Keywords: Energy efficiency. Passive strategies. Photovoltaics;

\section{INTRODUÇÃO}

Desde o acontecimento das crises energéticas, como a do petróleo, surgiu uma crescente preocupação em relação ao consumo energético mundial e ao uso de energias limpas, com o intuito de preservar os recursos ambientais. Dentro desse contexto de consumo, os edifícios são responsáveis pelo uso de cerca de $40 \%$ da energia primária mundial (International Energy Agency; Organization for Economic Co-operation and Development, 2009). 
Desde a antiguidade a construção civil existiu para atender as necessidades básicas do ser humano, a princípio sem a preocupação quanto as técnicas adotadas e seus impactos no meio ambiente (CORRÊA, 2009). Atualmente, esse contexto tem mudado, após as dificuldades e baixo crescimento, devido à falta de incentivo, o cenário construtivo encontrase em um novo ciclo de desenvolvimento, contribuindo para a economia nacional de forma expressiva. De acordo com Roth e Garcias (2009) o setor da construção ocupa posição de destaque com uma parcela significativa no Produto Interno Bruto (PIB). Como afirmam Holmes e Hacker (2007), o desafio atual dos profissionais da área da construção civil é projetar edificações de baixo consumo energético, de maneiras mais sustentáveis, e ainda assim, proporcionar conforto térmico aos seus usuários.

Diante disso, como cita Rodriguez-Ubinas et al. (2014), se torna imprescindível a construção de edifícios eficientes, ou seja, aqueles que fazem uso de estratégias passivas como sombreamento e orientação adequada, diminuindo assim, a demanda de refrigeração, iluminação e aquecimento, consequentemente atenuando o consumo de energia.

O melhoramento de um edifício energeticamente eficiente é denominado Edifício de Energia Zero - EEZ, segundo Thomas e Duff (2013). Os EEZ produzem através de geradores de energia renovável, toda a energia que é consumida no período de um ano, podendo ultrapassar essa demanda. Como exemplo disso existem as placas fotovoltaicas que geram energia a partir da obtenção de radiação solar, esse sistema possui a vantagem de gerar pouco impacto ambiental, assim como outras fontes de energia renovável (BRAGA et al., 2005).

Construções de baixo impacto ganham cada vez mais atenção, no entanto, não existe uma definição clara sobre os critérios para que uma construção se torne um EEZ. Sartori, Napolitano e Voss (2012) e Marszal et al. (2011) questionam esse ponto alegando que a falta de definição exata de critérios normativos e de desempenho deixam uma lacuna para que edifícios energeticamente ineficientes, com uma quantidade exagerada de placas solares, sejam classificados como EEZ, o que não é desejado, pois o sistema poderia se tornar economicamente inviável e com geração mal aproveitada de energia, mesmo sendo renovável. Torcellini et al. (2006) definiu as prioridades para um EEZ, o primeiro fator foi a questão da eficiência energética e depois a aplicação das fontes de energia renováveis.

Segundo Sartori, Napolitano e Voss (2012) há dois tipos de EEZ, os ligados à rede e os autônomos. Os primeiros são mais fáceis de serem alcançados, porém enfrentam o desafio de não sobrecarregarem a rede elétrica. $O$ ideal é que a produção seja principalmente em momentos de falta, como o horário de pico de consumo. Já os autônomos não correm esse risco, entretanto, se houver uma mudança abrupta e não predita no clima, o edifício pode não produzir a energia necessária para atingir o desempenho necessário (SARTORI; NAPOLITANO; VOSS 2012).

Deng et al. (2011) demonstram que a maioria dos projetos de EEZ possui deficiências e limitações, como a não possibilidade de reprodução exata de medidas passivas e do dimensionamento de geradores de energia renovável por causa do clima, velocidade do vento, incidência solar, estilo de vida e características locais. Tais incertezas e instabilidades evidenciam a necessidade de uma análise específica para cada projeto (ZHANG et al., 2016).

As estratégias de eficiência energética são mais individuais do que os meios de produção de energia renovável, por isso grande parte das pesquisas realizadas não citam quais medidas foram utilizadas. Os poucos que as mencionam falam principalmente da luz natural, dimensionamento e orientação adequada das aberturas, sombreamento, envoltória apropriada e ventilação natural (GIORDANO et al., 2015; AKSAMIJA, 2015; LI et al., 2013; KAPSALAKI et al., 2012).

Há a convergência dos EEZ serem obrigatórios no futuro. Na União Europeia o prazo é a partir de 2020 (European Parliament, 2011), e na Califórnia, nos Estados Unidos, o objetivo é tornar obrigatório para o setor residencial em 2020 e para o comercial em 2025 
(California Legislature, 2009). Já no Brasil entretanto, ainda falta consolidação das pesquisas voltadas a esse tipo de edifício, existindo lacunas, como por exemplo a necessidade de verificação e estudos de viabilidade técnica e econômica de implantação desse sistema.

A grande questão sempre levantada pelos profissionais da construção civil é até onde usar medidas de eficiência energética em termos de custo benefício (MARSZAL \& HEISELBERG, 2011). Dependendo do clima e região, o custo para melhorar a física de edificação com paredes duplas pode sair mais caro que o gasto com refrigeração e/ou aquecimento, por exemplo.

Portanto é essencial haver um equilíbrio e uma simulação adequada, visando as melhores estratégias para ser aplicada em cada caso específico. Deng et al. (2011) ressalta que a maioria dos projetos não podem ser compartilhados por causa do clima e características culturais. Destaca também, que a maioria dos projetos não analisam a viabilidade econômica.

Dessa forma, a avaliação da viabilidade econômica do edifício torna-se necessária, devido a necessidade de expor se é viável ou não economicamente a produção desse tipo de edificação. No Brasil existem apenas as pesquisas desenvolvidas por Pacheco (2013), Didoné, Wagner e Pereira (2014) e Guarda et al. (2020). Os dois primeiros abordam apenas duas cidades e o último, uma. Portanto, nota-se a ausência de informações necessárias para o desenvolvimento de uma referência que atenda a extensão territorial do Brasil. Os poucos climas pesquisados associados à incipiência de pesquisas que verificam a viabilidade econômica por meio do custo do ciclo de vida embasam este artigo, considerando uma cidade no centro do cerrado brasileiro.

\subsection{Objetivo}

O objetivo desse trabalho é propor um projeto e avaliar a viabilidade econômica da implantação de uma Casa de Energia Zero de baixa renda, para popularização em regiões de clima quente, com auxílio de diferentes envoltórias e estratégias bioclimáticas.

\section{MATERIAIS E MÉTODOS}

\subsection{Objeto de estudo}

A cidade de Cuiabá no estado de Mato Grosso foi escolhida como local para este objeto de estudo por possuir elevadas temperaturas médias anuais e altas radiações o ano todo, com médias de $210 \mathrm{Wh} / \mathrm{m}^{2}$ (PROJETEEE, 2018). Além disso, o software utilizado já possui o arquivo climático integrado ao seu sistema, facilitando a análise dos dados de clima.

Para alcançar o objetivo descrito acima foi primeiramente escolhido um projeto de baixo padrão de habitação de interesse social, com aproximadamente $60 \mathrm{~m}^{2}$ de área construída. Deu-se preferência ao estudo de viabilidade em uma moradia de baixo padrão devido à alta disseminação destas no território nacional e, também por notar-se a necessidade proporcionar maior conforto nesta tipologia de edificações.

\subsection{Simulação computacional}

A partir da escolha da edificação foram realizadas adequações no projeto original, utilizando-se estratégias passivas com aplicação de estratégias de eficiência energética, como: sombreamento por meio de beirais prolongados, uso de iluminação natural, adequação da área ideal para janelas; orientação adequada e resistência térmica da envoltória melhorada. Como a edificação foi analisada de forma isolada não foi levado em consideração o entorno, seja de outras casas ou vegetação. Considerou-se a orientação adequada nas aberturas da edificação e um beiral para o sombreamento por questões econômicas, tendo assim, um menor ganho de carga térmica e consequentemente redução do consumo de energia. 
Após as definições básicas do objeto de estudo, definiu-se cinco cenários de estudo, descritos no item 2.1. Tais cenários foram denominados por meio da sigla CEZ - Casa de Energia Zero, após a aplicação da geração de energia por meio dos painéis fotovoltaicos. Para essa pesquisa foi adotado o tipo de sistema fotovoltaico ligado à rede, pois além de possuírem a capacidade de fácil alcance, essa ligação já está regulamentada pela RN 482. (ANEEL, 2012)

Para as simulações de desempenho e eficiência energéticas foi utilizado o software DesignBuilder, desenvolvido pela empresa britânica DesignBuilder. O programa possui interface gráfica bastante intuitiva e amigável que permitem a utilização de forma eficiente dos parâmetros como ventilação natural, iluminação natural, dividido em abas nomeadas com cada item construtivo, e ainda, conta com um banco de dados com diferentes materiais para construção, diferenciando-se do software de origem, o EnergyPlus, que é um programa baseado em programação de scripts e inserção de códigos. De acordo com Lopes (2012), o DesignBuilder utiliza os modelos matemáticos provenientes do EnergyPlus e, também, contém um acervo de arquivos climáticos incluindo mais de 2.000 cidades com validação comprovada (LEÃO, 2011). Com a modelagem das CEZ e simulação da eficiência energética pode-se verificar qual cenário apresenta menor consumo de energia, sendo, dessa forma, avaliadas, quais as soluções apresentaram-se mais eficiente. Os schedules de horários de funcionamento foram estabelecidos de acordo com o regulamento de etiquetagem brasileiro (INMETRO, 2012). O software gera o output de consumo e a casa com melhor desempenho foi a escolhida e testada. Para a verificação do desempenho térmico do modelo com menor consumo foi realizada a classificação da eficiência energética por meio da simulação horária e análise das temperaturas obtidas na edificação, de acordo com as instruções do INMETRO (2012) (Figura 1) para a zona bioclimática 7, na cidade de Cuiabá-Mato Grosso, Brasil.

Figura 1 - Equivalente numérico da envoltória em função dos GHr na zona bioclimática 7

\begin{tabular}{c|c|c}
\hline Eficiência & EqNumEnvAmb & Condição \\
\hline A & 5 & $\mathrm{GH}_{\mathrm{R}} \leq 12.566$ \\
\hline B & 4 & $12.566<\mathrm{GH}_{\mathrm{R}} \leq 18.622$ \\
\hline C & 3 & $18.622<\mathrm{GH}_{\mathrm{R}} \leq 24.679$ \\
\hline D & 2 & $24.679<\mathrm{GH}_{\mathrm{R}} \leq 30.735$ \\
\hline E & 1 & $\mathrm{GH}_{\mathrm{R}}>30.735$ \\
\hline
\end{tabular}

Fonte: Adaptado INMETRO (2012).

\subsection{Avaliação financeira}

Os custos são considerados para o ciclo de vida da construção, sendo assim, os custos foram calculados em duas partes: o investimento inicial e o custo da energia. Para isso, foi utilizado o Custo Unitário Básico de Construção $\left(\mathrm{CUB} / \mathrm{m}^{2}\right)$ como referência, que variam de acordo com a economia de cada estado. A responsabilidade de calcular o $\mathrm{CUB} / \mathrm{m}^{2}$ é dos Sindicatos da Indústria da Construção e para esta pesquisa foi utilizado como base o valor de $\mathrm{R} \$ 1,403.17 / \mathrm{m}^{2}$, valor médio do ano de 2017 (CBIC, 2017). Sendo assim, o investimento inicial conta com o valor gasto com a construção como um todo e os geradores de energia no caso da CEZ. O custo da energia é uma variável que entrará apenas na casa padrão pois na CEZ os geradores de energia renovável devem prover energia considerando o balanço anual. 
Ao fazer uma análise financeira das soluções de eficiência energética propostas para os cenários de estudo, adotou-se uma casa padrão como referencial, sendo denominada Casa Padrão. A viabilidade foi calculada por meio do Valor Presente Líquido (VPL,) que tem como finalidade determinar o valor com todos os descontos e investimentos no momento inicial. A viabilidade financeira de um investimento é estudada através de um prazo estipulado por um investidor, onde se estima saber se o esforço aplicado vale mais do que uma aplicação desse mesmo valor aplicado no mercado com Taxas Mínimas de Atratividade - TMA (HIRSCHFELD, 2000). Tal viabilidade foi verificada através do tempo de repagamento, ou seja, quando os benefícios compensam o valor do investimento com a instalação e manutenção das soluções propostas, utilizando para isso o VPL, conforme a equação 1.

O período considerado para avaliação do VPL foi de 25 anos, o fluxo de caixa foi o consumo de energia anual da Casa Padrão e a Taxa Mínima de Atratividade (TMA) foi baseada na taxa do Sistema Especial de Liquidação e de Custódia - Selic, calculada pelo Banco Central do Brasil de 8,25\%.

$$
V P L=\sum_{0}^{n} \frac{C}{(1+i)^{n}}
$$

Onde:

VPL $=$ Valor presente líquido

$\mathrm{n}=$ número de períodos envolvidos

$\mathrm{C}=$ valores envolvidos no fluxo de caixa

$\mathrm{i}=$ taxa mínima de atratividade

\subsection{Cenários de estudo}

\subsubsection{Modelo referencial - Casa Padrão}

Para este modelo foi considerada uma casa de baixo padrão com $56,50 \mathrm{~m}^{2}$ de área construída, conforme a Figura 2, na Tabela 1 as aberturas são detalhadas. Considerou-se que a Casa Padrão continha dois quartos com $10,65 \mathrm{~m}^{2}$ cada, sala e cozinha conjugadas com 22,40 $\mathrm{m}^{2}$, banheiro com 4,05 $\mathrm{m}^{2}$ e áreas externas contabilizando $8,75 \mathrm{~m}^{2}$. O modelo segue a tipologia de casas de baixo padrão da região de Cuiabá, algumas mudanças foram realizadas buscando maior desempenho térmico e para futura instalação e manutenção dos sistemas fotovoltaicos, nesse e nos outros modelos. As aberturas são voltadas para leste e oeste considerando o pior caso de insolação sem sombreamento. As características físicas incluem paredes simples de tijolo furado com revestimento de argamassa, resultando em uma transmitância térmica de 3,19 W/m.K (Tabela 2) laje de concreto impermeabilizada com transmitância térmica de $1,69 \mathrm{~W} / \mathrm{m}^{2} . \mathrm{K}$ na cobertura e vidro simples comum de $3 \mathrm{~mm}$ nas janelas.

Tabela 1 - Tipologia das aberturas

\begin{tabular}{c|c|c|c}
\hline Janelas & Sala/Cozinha & Quarto 1 e 2 & Banheiro \\
\hline Dimensões $(\mathrm{m})$ & $3.90 \times 1.10$ & $2.30 \times 1.10$ & $0.80 \times 0.50$ \\
\hline Area $\left(\mathrm{m}^{2}\right)$ & 4.29 & 2.53 & 0.40 \\
\hline
\end{tabular}

Figura 2 - Planta baixa de referência 
Domingos, Renata; Gabriel, Elaise; Proposta de modelo Casa de Energia Zero (CEZ) de baixo padrão para Cuiabá-MT com avaliação da viabilidade econômica. E\&S - Engineering and Science, 2020, 9:2.

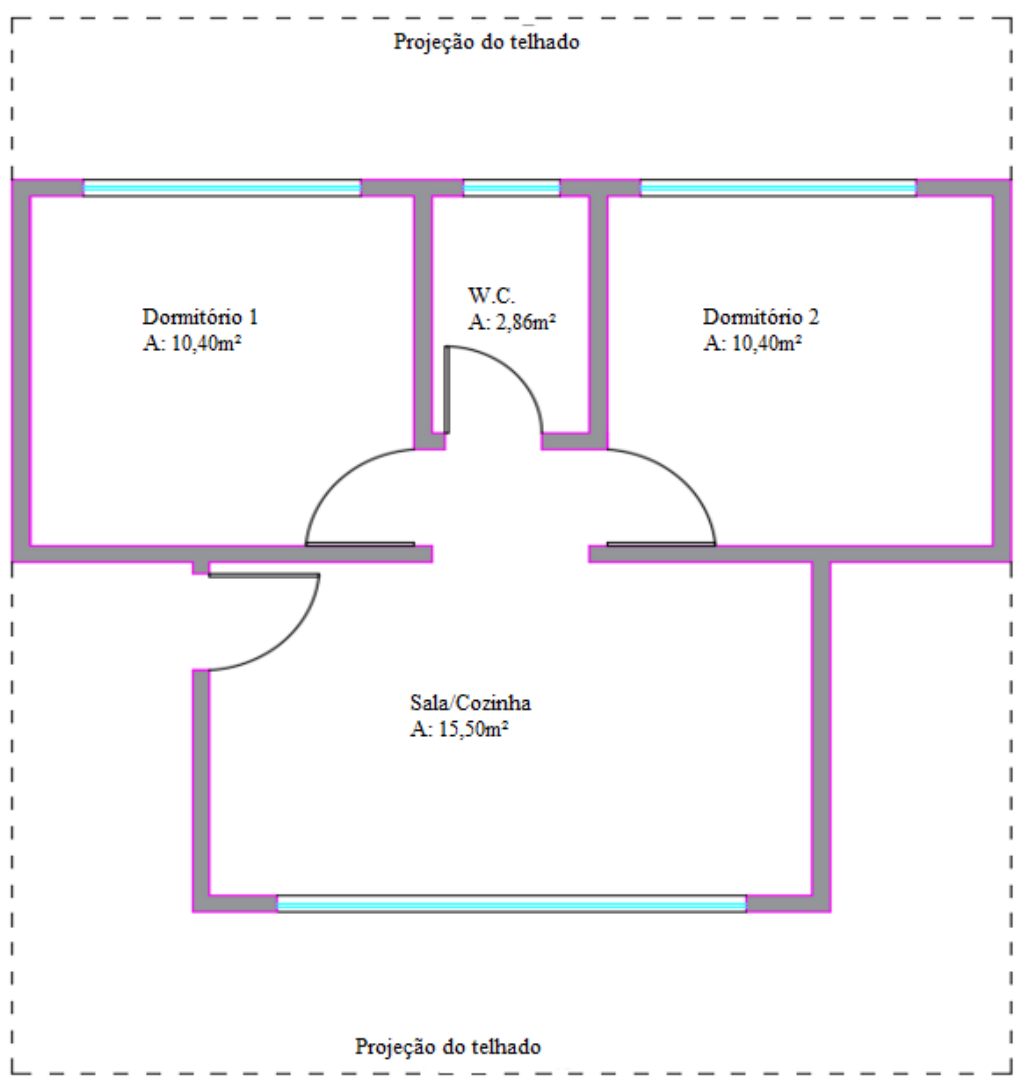

Tabela 2 - Propriedades termofísicas dos materiais construtivos CEZ 1

\begin{tabular}{c|c|c|c|c|c|c}
\hline \multirow{2}{*}{ Envoltória } & Composição & $\begin{array}{c}\text { Espessura } \\
(\mathrm{cm})\end{array}$ & $\begin{array}{c}\text { Absortância } \\
(\alpha)\end{array}$ & $\begin{array}{c}\text { Calor } \\
\text { Específico }(\mathrm{c}) \\
(\mathrm{J} / \mathrm{Kg} . \mathrm{K})\end{array}$ & $\begin{array}{c}\text { Condutividade } \\
\text { Térmica }(\gamma) \\
\left(\mathrm{Wm}^{-2} \mathrm{~K}\right)\end{array}$ & $\begin{array}{c}\text { Densidade } \\
(\rho)\left(\mathrm{Kg} / \mathrm{m}^{3}\right)\end{array}$ \\
\hline \multirow{2}{*}{ Paredes } & Argamassa & 2.00 & 0.30 & 1000 & 1.15 & 2000 \\
\cline { 2 - 7 } & Bloco cerâmico & 10.00 & 0.85 & 920 & 0.90 & 1600 \\
\cline { 2 - 7 } & Argamassa & 2.00 & 0.30 & 1000 & 1.15 & 2000 \\
\hline \multirow{2}{*}{ Cobertura } & Concreto & 10.00 & 0.30 & 1000 & 1.75 & 2400 \\
\hline
\end{tabular}

\subsubsection{Cenário $C E Z-2$}

O CEZ-2 possui as mesmas características do modelo anterior com diferencial para a resistência térmica da envoltória. Nesse modelo considerou-se a utilização de paredes duplas de tijolos furados na orientação oeste, com o intuito de diminuir a carga térmica através de uma menor transmitância da parede, que é de $2,37 \mathrm{~W} / \mathrm{m}^{2} . \mathrm{K}$ (Tabela 3). Os painéis fotovoltaicos foram redimensionados de acordo com a nova necessidade e o mesmo se aplica aos modelos abaixo. 
Domingos, Renata; Gabriel, Elaise; Proposta de modelo Casa de Energia Zero (CEZ) de baixo padrão para Cuiabá-MT com avaliação da viabilidade econômica. E\&S - Engineering and Science, 2020, 9:2.

Tabela 3 - Propriedades termofísicas dos materiais construtivos CEZ 2

\begin{tabular}{c|c|c|c|c|c|c}
\hline \multirow{2}{*}{ Envoltória } & Composição & $\begin{array}{c}\text { Espessura } \\
(\mathrm{cm})\end{array}$ & $\begin{array}{c}\text { Absortância } \\
(\alpha)\end{array}$ & $\begin{array}{c}\text { Calor } \\
\text { Específico }(\mathrm{c}) \\
(\mathrm{J} / \mathrm{Kg} . \mathrm{K})\end{array}$ & $\begin{array}{c}\text { Condutividade } \\
\text { Térmica }(\gamma) \\
\left(\mathrm{Wm}^{-2} \mathrm{~K}\right)\end{array}$ & $\begin{array}{c}\text { Densidade } \\
(\rho)\left(\mathrm{Kg} / \mathrm{m}^{3}\right)\end{array}$ \\
\hline \multirow{2}{*}{ Paredes } & Argamassa & 2.00 & 0.30 & 1000 & 1.15 & 2000 \\
\cline { 2 - 7 } & Bloco cerâmico & 20.00 & 0.85 & 920 & 0.90 & 1600 \\
\cline { 2 - 7 } & Argamassa & 2.00 & 0.30 & 1000 & 1.15 & 2000 \\
\hline \multirow{2}{*}{ Cobertura } & Concreto & 10.00 & 0.30 & 1000 & 1.75 & 2400 \\
\hline
\end{tabular}

\subsubsection{Cenário CEZ - 3}

Para o CEZ-3 foram adotadas as mesmas propriedades do CEZ-1, entretanto a parede dupla de tijolos furados foi aplicada para a orientação sul com o objetivo de verificar a viabilidade de instalação dessa técnica em diferentes cenários levando em conta o consumo de energia reduzido por conta dessa aplicação.

\subsubsection{Cenário CEZ - 4}

Da mesma forma que os anteriores, CEZ-4 possui as mesmas características construtivas que o CEZ-1, contudo para esse exemplo foi aplicado um sistema construtivo com poliestireno expandido - EPS (Figura 2) na fachada oeste, totalizando uma transmitância de $0,96 \mathrm{~W} / \mathrm{m}^{2}-\mathrm{K}$ (Tabela 4 ).

Tabela 4 - Propriedades termofísicas dos materiais construtivos CEZ 4

\begin{tabular}{c|c|c|c|c|c|c}
\hline \multirow{5}{*}{ Envoltória } & Composição & $\begin{array}{c}\text { Espessura } \\
(\mathrm{cm})\end{array}$ & $\begin{array}{c}\text { Absortância } \\
(\alpha)\end{array}$ & $\begin{array}{c}\text { Calor } \\
\text { Específico }(\mathrm{c}) \\
(\mathrm{J} / \mathrm{Kg} . \mathrm{K})\end{array}$ & $\begin{array}{c}\text { Condutividade } \\
\text { Térmica }(\gamma) \\
\left(\mathrm{Wm}^{-2} \mathrm{~K}\right)\end{array}$ & $\begin{array}{c}\text { Densidade } \\
(\rho)\left(\mathrm{Kg} / \mathrm{m}^{3}\right)\end{array}$ \\
\hline \multirow{5}{*}{ Paredes } & Argamassa & 2.00 & 0.30 & 1000 & 1.15 & 2000 \\
\cline { 2 - 7 } & Bloco cerâmico & 10.00 & 0.85 & 920 & 0.90 & 1600 \\
\cline { 2 - 7 } & EPS & 3.00 & 0.20 & 1420 & 0.04 & 35 \\
\cline { 2 - 7 } & Argamassa & 2.00 & 0.30 & 1000 & 1.15 & 2000 \\
\hline \multirow{2}{*}{ Cobertura } & Concreto & 10.00 & 0.30 & 1000 & 1.75 & 2400 \\
\hline
\end{tabular}


Domingos, Renata; Gabriel, Elaise; Proposta de modelo Casa de Energia Zero (CEZ) de baixo padrão para Cuiabá-MT com avaliação da viabilidade econômica. E\&S - Engineering and Science, 2020, 9:2.

Figura 3 - Sistema construtivo com tijolo e EPS

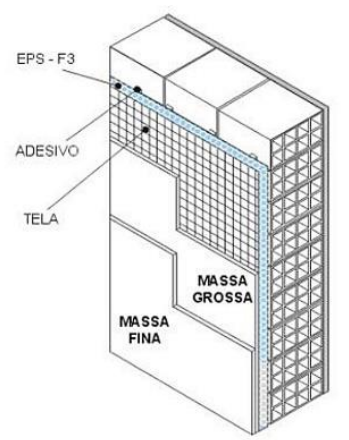

Fonte: Santos et al. (2013).

\section{RESULTADOS}

Com base nas simulações realizadas chegou-se ao consumo de cada cenário analisado. O cenário com o menor consumo energético foi o CEZ-4, com a característica construtiva de sistema de EPS na parede oeste. Dessa forma ele foi tomado como o protótipo ideal de baixo padrão, dentre os modelados, considerando as características climáticas da cidade de CuiabáMT.

\subsection{Viabilidade Técnica e Financeira}

Diante disso, tomando o CEZ-4 como ideal, realizou-se um cálculo de aumento percentual de consumo energético comparando com os demais cenários estudados, levando em consideração os padrões do RTQ-R. O CEZ Referência consumiu cerca de 38,9\% a mais de energia do que o modelo ideal. Os CEZ-1, CEZ-2, CEZ-3 consumiram respectivamente $0,57 \%, 0,23 \%$ e $0,52 \%$ a mais que o CEZ-4.

Percebeu-se que não houve acentuada diferença entre os cenários 2,3 e 4, já que os mesmos possuem medidas de eficiência energética aplicadas como sombreamento, iluminação natural, área ideal de janela e orientação adequada de aberturas. Tais resultados demonstraram que o investimento na resistência térmica da envoltória pode não ser viável financeiramente se comparado ao investimento das outras estratégias, principalmente, as de projeto, que não tem custo adicional. Por isso, é interessante a adoção dessas estratégias antes das que requerem investimento financeiro. No entanto a premissa para um CEZ é o edifício com menor consumo por isso o edifício escolhido como ideal foi o CEZ-4. Ao se calcular o VPL obteve-se que o período de retorno para o investimento do CEZ-4 foi de 6,94 anos. A Figura 3 demonstra como as melhorias nas envoltórias tiveram baixo impacto no período de retorno. 
Domingos, Renata; Gabriel, Elaise; Proposta de modelo Casa de Energia Zero (CEZ) de baixo padrão para Cuiabá-MT com avaliação da viabilidade econômica. E\&S - Engineering and Science, 2020, 9:2.

Figura 4 - Período de retorno dos cenários estudados

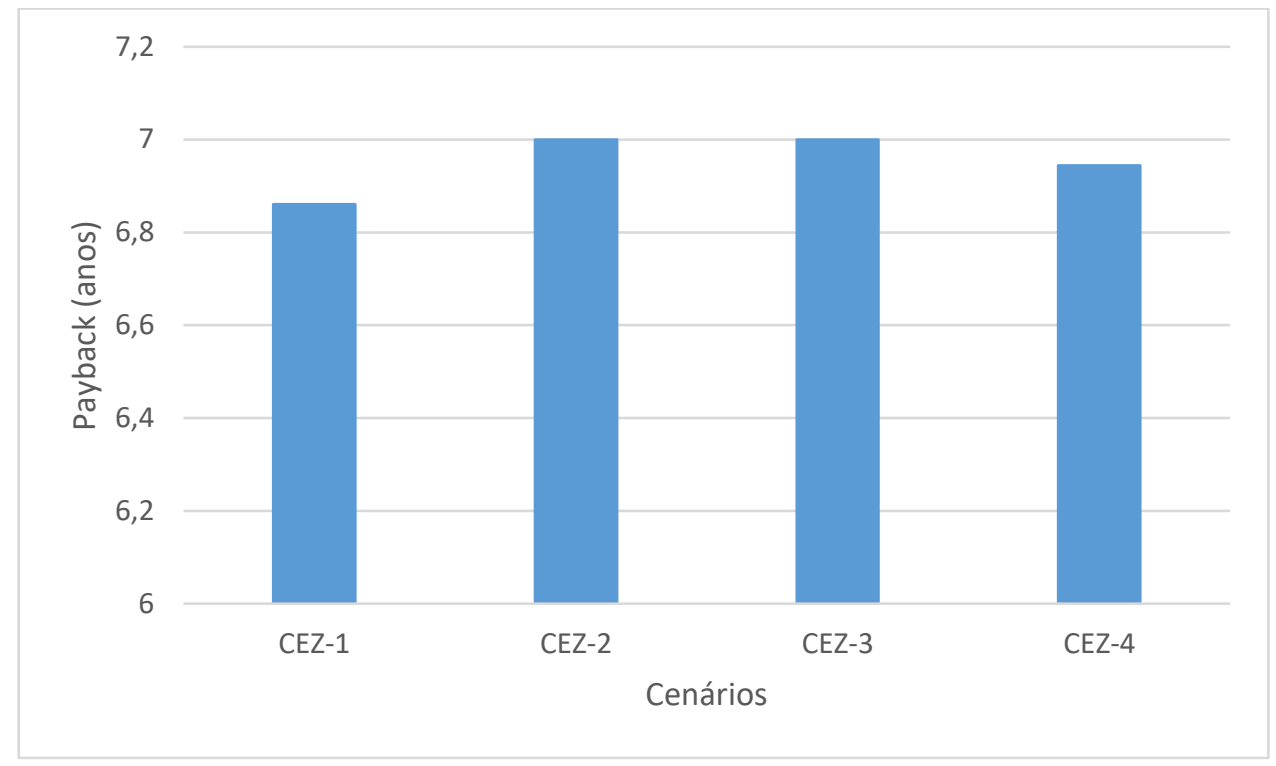

\subsection{Classificação da Eficiência Energética}

Para verificar se a modelagem do CEZ-4 era de fato eficiente energeticamente e cumpria com os requisitos de conforto estabelecidos pela Portaria $n^{\circ} 499$ do INMETRO (2012), foi realizada a etiquetagem por necessidade de graus-horas de resfriamento. Cada ambiente de permanência prolongada, quartos e sala/cozinha, foi simulado de forma separada (Figura 4) de acordo com os requisitos da Portaria (Quadro 1). Depois da simulação foi feita uma ponderação geral dos resultados obtidos para cada ambiente, tendo o resultado final da envoltória da casa a classificação " $\mathrm{A}$ " em eficiência, necessitando de 11.841,42 graus-horas de resfriamento em toda casa. Demonstrando como o modelo proposto está dentro do esperado de uma CEZ que é uma casa eficiente com micro geração de energia.

Figura 5 - Classificação de eficiência energética por ambiente

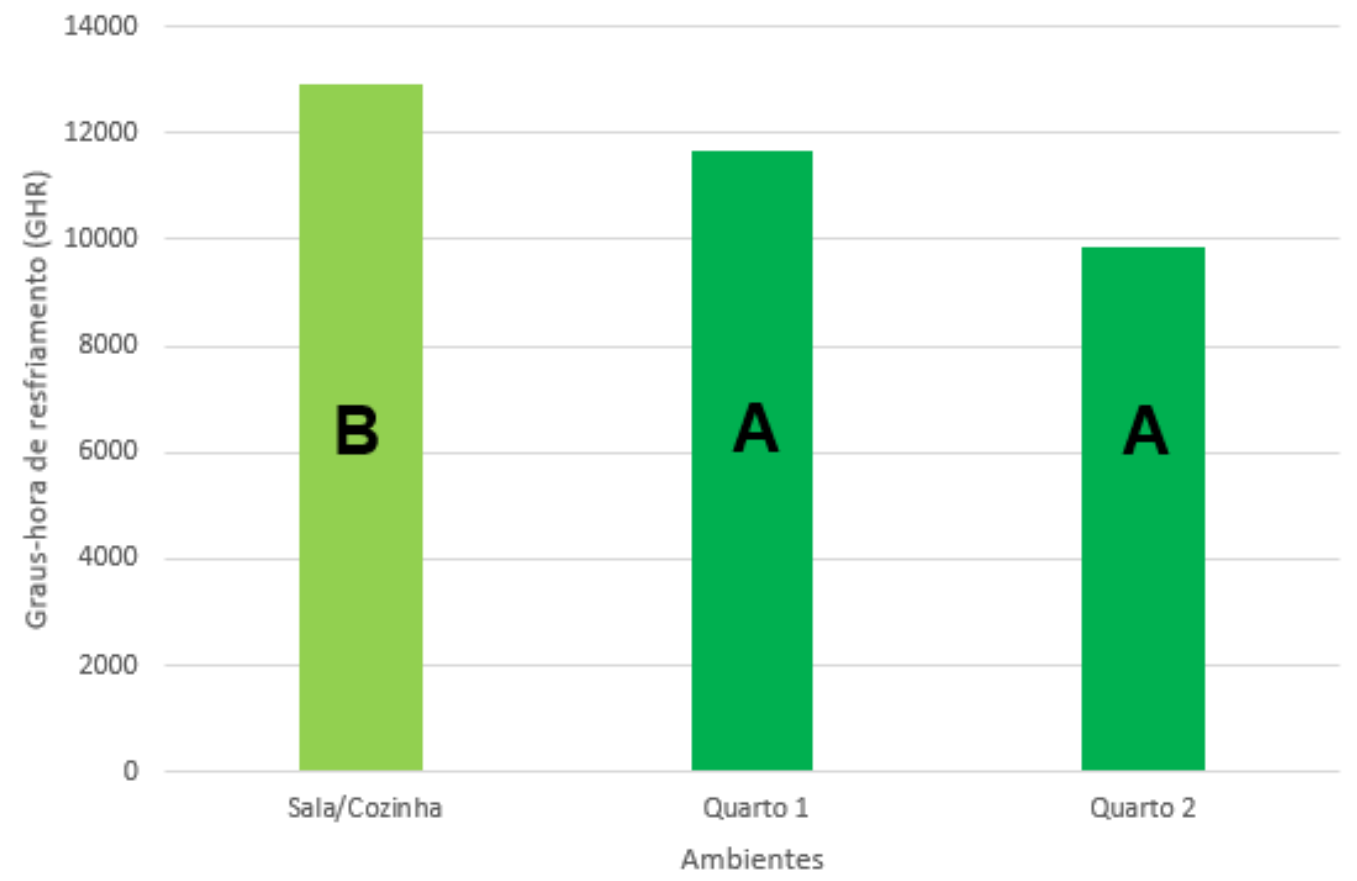


Domingos, Renata; Gabriel, Elaise; Proposta de modelo Casa de Energia Zero (CEZ) de baixo padrão para Cuiabá-MT com avaliação da viabilidade econômica. E\&S - Engineering and Science, 2020, 9:2.

\section{CONCLUSÃO}

As simulações realizadas nos diferentes cenários de estudo, por meio da modelagem utilizando o software DesignBuilder, demonstraram a importância de avaliação das estratégias passivas a serem aplicadas na edificação antes da adequação da sua estrutura física, quando se visa melhorar seu desempenho de consumo energético. Sendo assim, o estudo realizado evidencia a necessidade da avaliação das possíveis soluções a serem adotadas para redução de consumo de energia desde as fases de concepção de projeto e de planejamento de uma obra, como afirma Lamberts et al. (2011). Essas medidas, caso adotadas durante a elaboração do projeto, podem resultar uma diminuição do consumo energético da edificação e melhor aproveitamento do desempenho dos equipamentos de geração de energia.

Considerando a diferença de consumo de energia elétrica dos cenários estudados, o CEZ-4 apresentou um consumo aproximadamente 38\% inferior ao CEZ-Referência e resultou em um retorno do investimento em aproximadamente 7 anos, período de tempo de retorno bem abaixo do tempo de vida de uma placa fotovoltaica (25 anos). Dessa maneira é valido afirmar que o trabalho traz subsidio para um investimento nessa configuração construtiva, podendo ser até mesmo por meio de políticas públicas, podendo ser implementado em programas federais como o Minha Casa Minha Vida.

Para futuras avaliações podem ser aplicadas outras estratégias de sombreamento, a consideração do entorno, o uso de vegetação e outros fatores que podem influenciar no conforto térmico e, consequentemente no consumo energia da edificação e na sua viabilidade econômica de implementação dos sistemas de geração de energia alternativa.

\section{REFERÊNCIAS}

ADHIKARI, R.S.; ASTE, N.; DEL PERO, C.; MANFREN, M. Net Zero Energy Buildings: Expense or Investment? Energy Procedia. v. 14, p.1331 - 1336, 2012. https://doi.org/10.1016/j.egypro.2011.12.1097

AGÊNCIA NACIONAL DE ENERGIA ELÉTRICA (ANEEL). Resolução normativa 482. Brasil, 2012.

AKSAMIJA, A. Regenerative Design of Existing Buildings for Net-Zero Energy Use. Procedia Engineering. v. 118, p.72 - 80, 2015. https://doi.org/10.1016/j.proeng.2015.08.405

ALAJMI, A.; ABOU-ZIYAN, H.; GHONEIM, A. Achieving annual and monthly net-zero energy of existing building in hot climate. Applied Energy. v. 165, p.511-521, 2016. https://doi.org/10.1016/j.apenergy.2015.11.073

BRAGA, B.; HESPANHOL, I.; CONEJO, J. G. L.; MIERZWA, J. C.; BARROS, M. T. L.; SPENCER M.; PORTO, M.; NUCCI, N.; JULIANO, N.; EIGER, S. Introdução à Engenharia Ambiental. 2. ed. São Paulo: Pearson Prentice Hall, 2005. 332 p.

CALIFORNIA LEGISLATURE. Assembly Bill no. 212. AB 212, 2009. Disponível em: $<$ http://www.leginfo.ca.gov/pub/09-10/bill/asm/ab_0201-

0250/ab_212_cfa_20090413_093432_asm_comm.html> Acesso em 28 de abr. 2016.

CBIC. CÂMARA BRASILEIRA DA INDÚSTRIA DA CONSTRUÇÃO. O Custo Unitário Básico. 2017. Disponível em: 〈http://www.cub.org.br/saiba-mais> Acesso em 25 de set. 2017. 
Domingos, Renata; Gabriel, Elaise; Proposta de modelo Casa de Energia Zero (CEZ) de baixo padrão para Cuiabá-MT com avaliação da viabilidade econômica. E\&S - Engineering and Science, 2020, 9:2.

CORRÊA, Lásaro Roberto. Sustentabilidade na Construção Civil. 2009. Monografia (Especialização). 70 f. Curso de Especialização em Construção Civil, Engenharia de Materiais e Construção, Escola de Engenharia da UFMG, Belo Horizonte, 2009.

DENG, S.; DALIBARD, A.; MARTIN, M.; DAI, Y.J.; EICKER, U.; WANG, R.Z. Energy supply concepts for zero energy residential buildings in humid and dry climate. Energy Conversion and Management. v. 52, p.2455-2460, 2011.

DENG, S.; WANG, R.Z.; DAI, Y.J. How to evaluate performance of net zero energy building e A literature research. Energy. v. 71, p.1-16, 2014.

DIDONÉ, E. L.; WAGNER, A.; PEREIRA, F. O. R. Estratégias para edifícios de escritórios energia zero no Brasil com ênfase em BIPV. Ambiente Construído, Porto Alegre, v. 14, n. 3, p. 27-42, jul./set. 2014.

EUROPEAN COUNCIL FOR AN ENERGY EFFICIENT ECONOMY. Steering Through the Maze \#2 Nearly Zero Energy Buildings: Achieving the EU 2020 Target. 2011. Disponível em:<http://www.eceee.org/buildings/Steering-2-zerobldgs.pdf> Acesso em 28 de abr. 2016.

GIORDANO, R.; SERRA, V.; TORTALLA, E.; VALENTINI, V.; AGHEMO, C. Embodied Energy and Operational Energy assessment in the framework of Nearly Zero Energy Building and Building Energy Rating. Energy Procedia 78: 3204 - 3209. 2015.

GONÇALVES, A.; NEVES, C.; COLÔBA, G.; NAKAGAWA, M.; MOTTA, R. R.; COSTA, R. P. Engenharia econômica e finanças. Rio de Janeiro: Elsevier, 2009.

GRAÇA, G. C.; AUGUSTO, A.; LERER, M. M.; Solar powered net zero energy houses for southern Europe: Feasibility study. Solar Energy 86: 634-646. 2012.

GUARDA, E. L. A. D.; DOMINGOS, R. M. A.; JORGE, S. H. M.; DURANTE, L. C.; SANCHES, J. C. M.; LEÃO, M.; CALLEJAS, I. J. A. The influence of climate change on renewable energy systems designed to achieve zero energy buildings inthe present: A case study in the Brazilian Savannah. Sustainable Cities and Society. 52: 101843. 2020,

HIRSCHFELD, H. Engenharia econômica e análise de custos. 7. ed. São Paulo: Atlas, 2000 .

HOLMES, M.J.; HACKER, J. N. Climate change, thermal comfort and energy: Meeting the design challenges of the 21st century. Energy and Buildings 39: 802-814. 2007.

INSTITUTO NACIONAL DE METROLOGIA, QUALIDADE E TECNOLOGIA INMETRO. Portaria $n^{\circ} 449$, de 25 de novembro de 2010. Aprovar o Regulamento Técnico da Qualidade - RTQ para o Nível de Eficiência Energética de Edificações Residenciais. Diário Oficial [da] República Federativa do Brasil, Rio de Janeiro, 17 jan. 2012.

INTERNATIONAL ENERGY AGENCY; ORGANISATION FOR ECONOMIC COOPERATION AND DEVELOPMENT. World Energy Outlook2009. Paris: IEA International Energy Agency; OECD, 2009. 
Domingos, Renata; Gabriel, Elaise; Proposta de modelo Casa de Energia Zero (CEZ) de baixo padrão para Cuiabá-MT com avaliação da viabilidade econômica. E\&S - Engineering and Science, 2020, 9:2.

KAPSALAKI, M.; LEAL, V.; SANTAMOURIS, M. A methodology for economic efficient design of Net Zero Energy Buildings. Energy and Buildings. v.55, p.765-778, 2012.

KWAN, Y.; GUAN, L. Design a Zero Energy House in Brisbane, Australia. Procedia Engineering. v.121, p.604-611, 2015.

LAMBERTS, R.; GHISI, E.; ABREU, A. L. P.; CARLO, J. C.; BATISTA，J. O.; MARINOSKI, D. L.; NARANJO, A. Desempenho térmico de edificações. 6 . ed. Florianópolis: [s.n.], 2011. 196 p.

LEÃO, M. Double skin façades in Brazil: energy and comfort analysis. 2011. Tese (Doutorado). 189 f. Curso de Arquitetura, Braunschweig University Of Technology, Braunschweig, 2011.

LI, D. H. W.; YANG, L.; LAM, J. C. Zero energy buildings and sustainable development implications - A review. Energy. v.54, p.1-10, 2013.

LOPES, F. S. M. E. Dimensionamento de um Permutador de Calor Terra- Ar e Avaliação do Impacte na Climatização de um Edifício. 2012. Dissertação (Mestrado em Engenharia Mecânica). 115f. Faculdade de Engenharia Mecânica do Instituto Superior Técnico de Lisboa, Portugal, 2012.

MARSZAL, A. J.; HEISELBERG, P. Life cycle cost analysis of a multi-storey residential Net Zero Energy Building in Denmark. Energy. v. 36, p.5600-5609, 2011

MARSZAL, A. J.; HEISELBERG, P.; BOURRELLE, J. S.; MUSALLC, E.; VOSSC, K.; SARTORI, I.; NAPOLITANO, A. Zero Energy Building - A review of definitions and calculation methodologies. Energy and Buildings. v. 43, p.971-979, 2011.

MINISTÉRIO DO MEIO AMBIENTE (Santa Catarina) (Comp.). Projeteee. Universidade Federal de Santa Catarina. Disponível em: <http://projeteee.mma.gov.br/dados-climaticos/>. Acesso em: 10 jun. 2018.

PACHECO, M. T. G. Ventilação natural e climatização artificial: crítica ao modelo superisolado para residência de energia zero em Belém e Curitiba. Tese (doutorado) 320f. Universidade Federal de Santa Catarina, Centro Tecnológico. Programa de Pós-Graduação em Engenharia Civil, Florianópolis, SC, 2013.

RODRIGUEZ-UBINAS, E.; RODRIGUEZ, S.; VOSS, K.; TODOROVIC, M. S. Energy efficiency evaluation of zero energy houses. Energy and Buildings. v. 83, p.23-35, 2014.

ROTH, G.; GARCIAS, C. M. Construção Civil e a Degradação Ambiental. Desenvolvimento em Questão, v. 7, n. 13, p. 111-128, 2009.

RUPP, R. F.; GHISI. E. Assessing window area and potential for electricity savings by using daylighting and hybrid ventilation in office buildings in southern Brazil. Simulation. v. 93(11), p.935-949, 2017. 
Domingos, Renata; Gabriel, Elaise; Proposta de modelo Casa de Energia Zero (CEZ) de baixo padrão para Cuiabá-MT com avaliação da viabilidade econômica. E\&S - Engineering and Science, 2020, 9:2.

SANTOS, C. G.; PALHARES, L. B.; DUARTE, R. O.; GONÇALVES, T. H. C; HORTA, M. M. A.; REIS, W. A.; STRAELH, R. V. POLIESTIRENO EXPANDIDO NA CONSTRUÇÃO CIVIL. Pós em Revista. Ed. 8. 2013.

SARTORI, I.; NAPOLITANO, A.; VOSS, K. Net zero energy buildings: A consistent definition framework. Energy and Buildings. v. 48, p.220-232, 2012.

SERGHIDES, D. K.; DIMITRIOU, S.; KATAFYGIOTOU, M. C.; MICHAELIDOU, M. ENERGY EFFICIENT REFURBISHMENT TOWARDS NEARLY ZERO ENERGY HOUSES, FOR THE MEDITERRANEAN REGION. Energy Procedia. v. 83, p.533 - 543, 2015.

TORCELlinI, P.; PLESS, S.; DERU, M.; CRAWLEY, D. Zero Energy Buildings: A Critical Look at the Definition, in: ACEEE Summer Stud, Pacific Grove, California, USA, 2006.

THOMAS, W. D.; DUFFY, J. J. Energy performance of net-zero and near net-zero energy homes in New England. Energy and Buildings. v. 67, p.551-558, 2013.

ZHANG, S.; HUANG, P.; SUN, Y. A multi-criterion renewable energy system design optimization for net zero energy buildings under uncertainties. Energy. v. 94, p.654-665, 2016. 